\title{
Reconciliación y paz. Un aporte desde la teología paulina*
}

\author{
Danilo Antonio Medina**
}

Recibido: 16 de enero de 2016 - Aprobado: 20 de marzo de 2016

\section{Resumen}

Este trabajo aborda desde la exégesis la histórica realidad nacional del conflicto, descubriendo en la comprensión bíblica de los conceptos de paz y reconciliación pistas para asumirla desde una ética cristiana. Aborda en primer lugar el Antiguo Testamento, en el que dada la vigencia de la ley del talión y una imagen de Yahvé que se nos presenta con frecuencia como la de un "vengador" (go'el), resulta poco factible encontrar elementos para defender la idea de la reconciliación, aunque muestra cómo en tal tradición, aquella encuentra su fuente y su sentido en Dios mismo. En Pablo, la reconciliación está en estrecha relación con el kerigma cristiano de la pasión, muerte y resurrección, vinculada así a un acontecimiento histórico que proyecta sus efectos tanto al ámbito de las relaciones interpersonales como al plano de las relaciones entre Dios y la humanidad, para desembocar en amor y disposición al perdón, pues anula la enemistad entre Dios y los hombres, o entre los grupos humanos. En cuanto a la paz, Pablo la vincula al concepto anterior, como resultado de la acción reconciliadora de Cristo en su misterio pascual, en el que se superan todos los prejuicios y esquemas que causan marginación y exclusión, en virtud de favoritismos de personas o de pueblos, siendo todos ahora un solo hombre nuevo, plenamente reconciliados y en armonía, con lógicas implicaciones en el campo social, desde las cuales el trabajo extrae pautas para la aplicación a la coyuntura histórica que lo motiva.

Palabras clave: teología paulina, paz, reconciliación, Colombia, víctimas. Minuto de Dios. Dirección postal: Calle 170 \# 8G-31, Bogotá, Colombia. Correo electrónico: danilomedina@sanpablo. com.co. 


\title{
Reconciliation and peace. A contribution from the Pauline theology perspective*
}

\author{
Danilo Antonio Medina**
}

\section{A bstract}

This paper discusses the local historical reality of conflict from the point of view of exegesis, unraveling clues based on the biblical understanding of the concepts of peace and reconciliation, and on Christian ethics in order to address it. First, the Old Testament is analyzed, since it is highly unlikely to find elements in it to defend the idea of reconciliation, given the force of the law of retaliation and a new image of the Lord that is frequently portrayed as an "avenger" (go'el), although, in such tradition, reconciliation may find its source and its sense in God Himself. Paul shows reconciliation as closely related to the Christian Kerygma of the passion, death and resurrection, and, therefore, related to a historical event that projects its effects on both interpersonal relationships and the relationships between God and humanity, to lead to love and readiness to forgive, because enmity between God and men, or between human groups is overridden. Regarding peace, Paul relates it to the concept mentioned above, as the result of the reconciling action of Christ in his paschal mystery, in which all prejudices and schemes that cause marginalization and exclusion, under favoritism of persons or peoples, are overcome since all of them are now only one new man, fully reconciled and in harmony, which has logical implications on the social field, from where this work gathers guidelines for application to the historical situation that generated it.

Keywords: Pauline theology; Peace; Reconciliation; Colombia; Victims.

Reflection paper. DOI: http://dx.doi.org/10.15332/s0120-8454.2016.0089.05

** Ph. D student in Biblical Theology in Universidad Pontifica Bolivariana Cebitepal. Degree in Theology from Pontificia Universidad Católica in Ecuador. Specialist in Sacred Scriptures from the Pontifical Biblical Institute in Rome. Teacher of Sacred Scriptures in Universidad San Buenaventura, Pontificia Universidad Javeriana and Corporación Universitaria Minuto de Dios, among others, as a member of the Asociación Colombiana de Escrituristas Católicos (ACEC), Address: Calle 170 \# 8G-31, Bogota, Colombia. Email: danilomedina@sanpablo.com.co. 


\title{
Réconciliation et paix. Un apport depuis la théologie paulinienne*
}

\author{
Danilo Antonio Medina*
}

\section{Résumé}

Ce travail aborde depuis l'exégèse l'historique réalité nationale du conflit armé, découvrant dans la compréhension biblique les concepts de paix et réconciliation, pistes pour l'assumer depuis une éthique chrétienne. Il aborde en premier lieu 1'Ancien Testament, dans lequel étant donné la validité de la loi du talion et une image de Yahvé qui nous est présenté fréquemment comme celui d'un "vengeur" (go'el), il résulte peut crédible de trouver des éléments pour défendre l'idée de réconciliation, même s'il montre comment dans cette tradition, celle-ci trouve sa source et son sens en Dieu même. Chez Pablo, la réconciliation est en étroite relation avec le kerigma chrétien de la passion, mort et résurrection, liée ainsi à un fait historique qui projette ses effets tant dans le domaine des relations interpersonnelles comme sur le plan des relations entre Dieu et l'Humanité, pour déboucher en amour et disposition au pardon, car cela annule la rivalité entre Dieu et les hommes, ou entre les groupes humains. En ce qui concerne la paix, Pablo la relie au concept antérieur, comme résultat de l'action de réconciliation du Christ dans son mystère de Pâques, dans lequel on dépasse tous les préjugés et schémas qui causent la marginalisation et l'exclusion, en vertu de favoritismes de personnes ou de peuples, en étant tous maintenant un seul homme nouveau, pleinement réconciliés et en harmonie, avec de logiques implications dans le domaine social, à partir desquelles le travail extrait des directives pour l'application à la conjoncture historique qui le motive.

Mots clés: théologie paulinienne, paix, réconciliation, Colombie, victimes.

Article de réflexion. DOI: http://dx.doi.org/10.15332/s0120-8454.2016.0089.05

Candidat au Doctorat en Théologie Biblique de I'Université Pontifica Bolivariana Cebitepal. Licencié en Théologie de I'Université Catholique d'Équateur. Spécialiste en Écriture Sacrée de I'Institut Biblique de Rome. Comme membre de la Asociación Colombiana de Escrituristas Católicos (ACEC), il est professeur en Écriture Sacrée, entre autres, à I'Université San Buenaventura, Université Javeriana et Corporation Universitaire Minuto de Dios. Adresse postale: Calle 170 \# 8G-31, Bogota, Colombie. E-mail: danilomedina@sanpablo.com.co. 


\section{Introducción}

Las últimas décadas de nuestra historia nacional, trágicamente marcadas por la violencia y el conflicto, nos convencen cada vez más de la imperiosa necesidad de encontrar caminos de reconciliación y de paz entre los colombianos. No deja de ser motivo de escándalo el constatar que un país, que en su gran mayoría se profesa creyente y cristiano, no haya logrado aún llevar al plano social y político las implicaciones éticas del compromiso de su fe. El presente trabajo no pretende hacer diagnósticos de la realidad, bastante conocida y sufrida, sino apelar a los fundamentos de nuestra fe cristiana, para encontrar en ella, y concretamente en la teología del apóstol Pablo, las motivaciones que puedan orientar y acompañar los procesos de reconciliación y paz que todos anhelamos, y, sobre todo, que la inmensa cantidad de víctimas reclama y merece.

\section{Trasfondo veterotestamentario}

Aparentemente, en el Antiguo Testamento (at) no sería muy fácil encontrar elementos para defender la idea de la reconciliación, habida cuenta de la vigencia de la así llamada ley del talión, que si bien representó un gran avance en el proceso de maduración del concepto de justicia, en cuanto establecía una necesaria equidad entre la ofensa y el modo de resarcirla, puede también llegar a justificar la venganza (cf. Ex 21, 23-25; Lv 24, 18-20; Dt 19, 21). Además, el Dios Yahvé del at con frecuencia se nos presenta bajo la imagen de un "vengador" (go'el), que pareciera estar de acuerdo con el desquite y la venganza (cf. Sal $17,13-15 ; 55,5-6.23 ; 75,8 ; 77,42-53)$. Sin embargo, encontramos algunos casos paradigmáticos de reconciliación entre personas, lo cual no era en todo caso lo más común. Por ejemplo, en la época de los patriarcas hallamos el episodio de la reconciliación entre Esaú y Jacob, luego de que este había arrebatado fraudulentamente los beneficios de la bendición de su padre Isaac, que le correspondían a su hermano (cf. Gn 33, 1-11).1

Son emotivas también las expresiones con las cuales se relata la reconciliación entre José y sus crueles hermanos (cf. Gn 45, 1-15), así como aquellas palabras que se reservan para elogiar la magnanimidad y sorprendente capacidad de perdón por parte de Moisés, cuando el pueblo que había ayudado a liberar de la esclavitud y que había acompañado y guiado por el desierto hacia la Tierra Prometida, en lugar de agradecerle, murmuraba constantemente contra él; y cuando incluso sus hermanos, Aarón y María, se llenaron de envidia contra él y lo criticaron (cf. Nm 12,1ss). También sobresale la piedad y capacidad de perdón que manifiesta David respecto del rey Saúl, luego de que este intentara matarlo (cf. 1 Sam 26, 1-25).

1 Acerca del proceso de reconciliación vivido por Jacob, no solo respecto de su hermano Esaú, sino incluso con Dios mismo, puede verse el breve y sencillo, pero muy acertado, comentario de Fischer (2010, pp. 1152-1154). 
No obstante lo anterior, en la tradición bíblica la reconciliación, como no podía ser de otra manera, encuentra su fuente y su motivación en Dios mismo. En otras palabras, la primera y fundamental reconciliación fue aquella que Dios quiso realizar con la humanidad, especialmente mediante el establecimiento de una alianza con su Pueblo elegido, en el monte Sinaí, justo cuando dicho pueblo venía murmurando contra Moisés y contra Él, acusándolos incluso de intenciones criminales (cf. Ex 17, 3; Nm 14, 1-4). Y a lo largo de toda la historia, esta va a ser la invitación permanente por parte de los profetas, a corresponder a la iniciativa divina, que quiere vivir en paz y armonía con la humanidad. De allí también brotará la necesidad de vivir reconciliados y en paz con los semejantes.

Precisamente en cuanto a la paz, no podemos decir que el at sea un manifiesto a su favor. De hecho, en una lectura superficial de sus libros y de su historia, más bien sobresalen el conflicto y las confrontaciones al interior del pueblo elegido, y entre este y otros pueblos. En ese contexto, la paz (shalom), se entiende en el más amplio sentido de bienestar, prosperidad, armonía, salud... En todo caso, su significado más profundo es ante todo religioso; la paz del at es un don de Dios, pues Él es paz y crea la paz, y bendice a su pueblo con la paz (cf. Jue 6, $24 ; \mathrm{Jb} 25,2$; Sal 35, 27; 122, 6). De este modo queda claro que la paz llega como bendición del Señor (Kittel, Friedrich y Bromiley, 2003, 207ss).

Cabe destacar, en fin, la contribución de los profetas, no sólo por su empeño en purificar los falsos conceptos de la paz y las falsas seguridades religiosas, recordando el compromiso ético y social de esa paz que quiere Yahvé (cf. Miq 3, 5ss; Jr 28, 1ss; 29, 11; Ez 13, 16; Is 48, 18; 54, 13), sino también por su apertura al horizonte escatológico en el cual tendrá cumplimiento la paz plena y universal que viene de Dios, pero que también implica el compromiso humano (cf. Is 2,2ss; 9, 5-7; Zac 9, 9-10) (Kittel et al., 2003, 207ss).

\section{En la teología paulina}

\section{Reconciliación ${ }^{2}$}

Si bien los términos usados por Pablo en sus cartas para referirse a la acción redentora de Cristo y sus consecuencias en el plano de la reconciliación y la paz social no alcanzan a recoger toda la riqueza y profundidad de su enseñanza al respecto, sí es conveniente y necesario acercarnos al sentido y uso que este término clave tiene, dado que en él se representan y expresan aspectos centrales de la teología paulina. El término reconciliación, tal como lo usa san Pablo, está en estrecha relación con el kerigma cristiano de la pasión, muerte y resurrección del Señor Jesús por nuestra salvación, dato que al vincular la reconciliación

2 Pablo usa varios verbos relacionados con la idea de reconciliar, que también puede significar 'cambiar' e 'intercambiar': avlla,ssw, avpalla,ssw, dialla,ssw, katalla,ssw, avpokalla,ssw, metalla,ssw, y sus sustantivos derivados. Estas formas verbales remiten a la idea de "hacer las paces", volver a la amistad, especialmente después de una guerra. Desde la perspectiva teológica, significan el regreso del hombre al favor y la intimidad con Dios, después del alejamiento provocado por el pecado (Fitzmyer, 1975, pp. 123-124). 
con un acontecimiento histórico, permite purificar este concepto de los matices gnósticos que tenía antes. Del mismo modo, los efectos que trae consigo la reconciliación en Cristo, en la enseñanza paulina, también son realidades concretas y verificables en la existencia del creyente: remisión de los pecados, nueva creación, justificación, redención, filiación, don del Espíritu, promesa de resurrección, etc. 3

El término reconciliación4 es usado por Pablo en sus cartas en varios contextos, tanto en el ámbito de las relaciones interpersonales como - especialmente - en el plano de las relaciones entre Dios y la humanidad; en ese sentido, podríamos afirmar con toda propiedad que Pablo revoluciona el concepto de reconciliación. Al recordar el trasfondo de la historia de la salvación, en cuyo contexto se interpretan las relaciones de la humanidad con Dios, en clave de Alianza, con mucha frecuencia se hace evidente la infidelidad humana respecto de dicha Alianza. Mientras Dios mantiene eternamente su fidelidad, el pueblo falla y ofende gravemente a Dios, reemplazándolo por los ídolos; pero llegada la plenitud de los tiempos, Dios envió a su Hijo al mundo de los hombres (cf. Gal 4, 4)

[...] para reconciliarnos consigo mismo y darles acceso a él (Rm 5, 1-2.8). Todas las promesas de Dios encuentran su "sí" en Cristo (2 Cor 1, 20). El misterio del evangelio ha manifestado este plan salvífico por el que Dios reconcilia todas las cosas (tanto los hombres como las demás criaturas) consigo mismo llevando a cabo la subordinación de todas las criaturas a Cristo, el kosmokrator. (Fitzmyer, 1975, 84)

Bajo esta perspectiva, cuando desde la lógica humana solo se podría esperar castigo, sucede algo inaudito; ahí está la gran novedad y cambio de paradigma, puesto de relieve por san Pablo: siendo Dios la parte ofendida, y siendo la parte más fuerte de los dos contrayentes de la alianza, Él mismo toma la iniciativa de perdonar y salvar por medio de su Hijo:

En efecto, cuando todavía estábamos sin fuerzas, en el tiempo señalado, Cristo murió por los impíos; — - en verdad, apenas habrá quien muera por un justo; por un hombre de bien tal vez se atrevería uno a morir-; mas la prueba de que Dios nos ama es que Cristo, siendo nosotros todavía pecadores, murió por nosotros... Si cuando éramos enemigos fuimos reconciliados con Dios por la muerte de su Hijo, ¡con cuánta más razón, estando ya reconciliados, seremos salvos por su vida! Y no solamente eso, sino que también nos gloriamos en Dios, por nuestro Señor Jesucristo, por quien hemos obtenido ahora la reconciliación. ( $\operatorname{Rm~5,6-11)~}$

3 Véase al respecto Martin (1999, p. 209). El autor se atreve a afirmar en su conclusión que todo lo que podamos afirmar acerca de las grandes ideas de la teología paulina confluyen en "reconciliación", como el título más apropiado y significativo que podemos darle al resultado final del comentario acerca del centro de la teología de Pablo.

$4 \quad$ Es muy elocuente el hecho de que de las catorce veces que aparecen los términos derivados del verbo reconciliar en el Nuevo Testamento, trece veces se encuentran en los escritos paulinos, y solo una vez fuera de él, en Mt 5, 24 (Luján, 1991, p. 487). 
La reconciliación es entendida como el principal fruto de la pasión, muerte y resurrección de Jesucristo, lo cual implica una radical restauración del ser humano en el estado de paz y unión con el Padre. Y es precisamente el Padre Dios el gran protagonista y agente de la reconciliación, pues aunque sea fundamental la intervención de Cristo (a través de su muerte: "por su sangre": Rm $5,9)$, Pablo insiste en atribuir la obra de la reconciliación al Padre, y es con Él con quien recuperamos la paz y la comunión, habiéndose superado o destruido la enemistad que lo impedía, gracias al sacrificio redentor de Cristo (cf. Rm 5, 10-11; Fitzmyer, 1975, pp. 122-123).

Esta nueva perspectiva presentada por el apóstol nos permite comprender las implicaciones que debe tener todo proceso de reconciliación cuando se inspira en el modelo de la ética cristiana. Dios es quien hace posible la reconciliación y quien nos hace experimentar su misericordia, con lo cual nos motiva y nos capacita para corresponder a dicha experiencia de gratuidad, ofreciendo el perdón y siendo agentes de reconciliación en nuestros ambientes familiares y sociales. Esta fue la experiencia de san Pablo, pues habiendo sido él mismo reconciliado con Dios, cuando era un perseguidor de la Iglesia, llega a sentir el peso de esta responsabilidad: ser embajador de la reconciliación, es decir, enviado por Dios para ejercer el ministerio de la reconciliación universal:

Y todo proviene de Dios, que nos reconcilió consigo por Cristo y nos confió el ministerio de la reconciliación. Porque en Cristo estaba Dios reconciliando al mundo consigo, no tomando en cuenta las transgresiones de los hombres, sino poniendo en nosotros la palabra de la reconciliación. Somos, pues, embajadores de Cristo, como si Dios exhortara por medio de nosotros. En nombre de Cristo les suplicamos: ¡reconcíliense con Dios! (2 Cor 5, 18-20)

Así pues, a la base de la enseñanza paulina sobre la reconciliación encontramos dos realidades inseparables: la necesidad de permanecer en amistad y comunión con Dios y la muerte redentora de Cristo, que restituye al ser humano la posibilidad de estar en paz con Dios y con sus semejantes. En efecto, la muerte en Cruz de Cristo hace posible la paz y la armonía cósmica y universal:

[...] para crear en sí mismo, de los dos [pueblos: judíos y paganos], un solo Hombre Nuevo, haciendo las paces, y reconciliar con Dios a ambos en un solo cuerpo, por medio de la cruz, dando en sí mismo muerte a la enemistad. (Ef 2, 15-16)

Los alcances de su acción redentora no son monopolio de un pueblo o de una raza; llegan a todas las personas que libre y voluntariamente deseen aceptarla: "Dios tuvo a bien hacer residir en Él toda la plenitud, y reconciliar por Él y para Él todas las cosas, pacificando, mediante la sangre de su cruz, los seres de la tierra y de los cielos" (Col 1, 19-20).

Las consecuencias de esta reconciliación actuada por Dios mediante el sacrificio de su Hijo descienden al plano concreto de la vida privada y pública. Con ello, 
el apóstol va delineando los rasgos típicos de una nueva ética personal, fruto de la reconciliación:

A ustedes, que en otro tiempo eran extraños y enemigos, por sus pensamientos y malas obras, los ha reconciliado ahora, por medio de la muerte en su cuerpo de carne, para presentarlos santos, inmaculados e irreprensibles delante de Él; con tal que permanezcan sólidamente cimentados en la fe, firmes e inconmovibles en la esperanza del Evangelio que oyeron... (Col 1, 21-23)

Pero también en el plano social, una nueva ética debe surgir para regir las relaciones interpersonales e internacionales: "Porque Él es nuestra paz: el que de los dos pueblos hizo uno, derribando el muro divisorio, la enemistad... Así pues, ya no son extraños ni forasteros, sino conciudadanos y familiares de Dios" (Ef 2, 14.19). A partir de estos parámetros generales, Pablo aplica su enseñanza a situaciones muy particulares y cotidianas (Fitzgerald, 2010, pp. 1161ss), por ejemplo, en el ámbito de la familia:

En cuanto a los casados, les ordeno, no yo sino el Señor: que la mujer no se separe del marido, mas en el caso de separarse, que no vuelva a casarse, o que se reconcilie con su marido, y que el marido no se divorcie de su mujer. (1 Cor 7, 10-11)

Y en el contexto de las comunidades cristianas, cuando surgen conflictos y litigios en su seno: "Ruego a Síntique y Evodia que se pongan de acuerdo en el Señor. También te ruego a Ti, Sícigo, compañero mío, que las ayudes..." (Filp 4, 2). Y la reconciliación debe desembocar en amor y disposición al perdón:

Pues si alguien me ha causado tristeza, no es a mí sólo a quien se la ha causado; sino en cierto sentido - para no exagerar- a todos ustedes. Bastante es para ese tal el castigo infligido por la mayoría; por lo que es mejor que lo perdonen más bien, y lo animen, no sea que se hunda en una excesiva tristeza. Les suplico, pues, que hagan prevalecer la caridad para con él. (2 Cor 2, 5-8)

\section{$\mathrm{Paz}^{5}$}

En cuanto al concepto $p a z^{6}$, el apóstol Pablo tiene también una enseñanza profunda y muy útil que ofrecernos. Dada la riqueza de la doble fuente cultural en la cual se formó (judía y grecorromana), Pablo recoge lo mejor de las dos

5 El término griego usado por Pablo para referirse al concepto de la paz no da oportunidad a confusiones, pues es uno solo: eivrh,nh. Sin embargo, debe discernirse su contenido cuidadosamente, pues como se verá más adelante, el sentido aquí atribuido no coincide del todo con el que le atribuía el Imperio romano, y ni siquiera con lo que entendía la tradición judía veterotestamentaria por la paz como Shalom: Alv'.

6 En el epistolario paulino, el término paz se usa 42 veces, mientras que al sumar las veces que aparece la palabra en los demás escritos del nt, se llega a 46; de modo que en Pablo se encuentran casi la mitad de las veces que aparece en todo el nt (42/88) (Luján, 1991, pp. 426-427). 
tradiciones, también en relación con el tema de la paz, pero estrechamente unido al tema de la reconciliación:

Reconciliación es el concepto paulino a través del cual se expresa el hecho que con la obra de Cristo queda abolida la enemistad entre Dios y los hombres, o entre los diversos bandos humanos, y se instauran relaciones pacíficas. Tal concepto se refiere a un estado objetivo de paz y no simplemente a una sensación de tranquilidad. (Porter, 1999, 1123)

Aunque del trasfondo judío Pablo pudo haber adoptado el sentido de paz, vinculado a la idea de bienestar exterior y material, probablemente haya que recurrir sobre todo al contexto griego, en el cual se entiende la paz como un tiempo o una condición libre de hostilidad o guerra: "Según la concepción griega, 'paz' es un término de relación, que designa un estado de bienestar objetivo que lleva a instaurar relaciones armoniosas entre pueblos o naciones" (Porter, 1999, 1125). Claro está que, como veremos, Pablo trasciende y enriquece con nuevas connotaciones y significados, eminentemente cristianos, el concepto de la paz.

Para acercarnos al sentido propiamente paulino de la paz hay que partir del hecho de que el Dios en quien creemos, tanto judíos como cristianos, es el Dios de la paz (cf. Filp 4, 9; 1 Tes 5, 28; 2 Tes 3, 16), y Él es amigo de la paz, "pues Dios no es un Dios de confusión, sino de paz" (1 Cor 14, 33). Pero esa paz de Dios encuentra su mejor expresión y su promotor más eficaz en Cristo. En Jesús se hace realidad el anhelado propósito de tener la paz verdadera, aquella que ni los ejércitos romanos pudieron garantizar, ni tampoco las tradiciones y leyes del pueblo judío. La convicción de Pablo no podía ser más clara ni más contundente: ¡Cristo es nuestra paz! (Ef 2,14); en Él se cumplieron todas las promesas de salvación, y por eso mismo es en Él que se encuentra el camino de la paz verdadera. La paz cristiana se identifica con la salvación, como bien lo hace notar el propio apóstol:

Habiendo, pues, recibido de la fe la justificación, estamos en paz con Dios, por nuestro Señor Jesucristo, por quien hemos obtenido también, mediante la fe, el acceso a esta gracia en la cual nos hallamos, y nos gloriamos en la esperanza de la gloria de Dios. (Rm 5, 1-2)

Se entiende por qué el apóstol no solo afirma que Dios es un Dios de paz, y que Cristo es nuestra paz, sino que también advierte que la paz es atributo y don de Dios y de Cristo: "Y la paz de Dios, que supera toda inteligencia custodiará sus corazones y sus mentes en Cristo Jesús" (Filp 4, 7); “Y que la paz de Cristo reine en sus corazones, pues a ella han sido llamados formando un solo cuerpo" (Col 3, 15). Para Pablo, la paz es un don de Dios, estrechamente unido al misterio pascual de Cristo. Por eso, en definitiva, más que un ambiente social o una actitud interior, la paz es una persona: es Cristo mismo. Al resultar vencedor del pecado y de la muerte, Cristo nos obtuvo la paz. Por eso es que Pablo insiste en que el Evangelio que él predica por el mundo entero, siendo el Evangelio del Cristo muerto y resucitado por nuestra salvación, es al mismo tiempo el Evangelio de la paz: "Pónganse en pie, ceñida su cintura con 
la verdad y revestidos de la justicia como coraza, calzados los pies con el celo por el Evangelio de la paz..." (Ef 6, 14-15).

La paz se presenta, de este modo, como el resultado de la acción reconciliadora de Cristo en su misterio pascual: con su muerte y resurrección, Él derribó los muros de división, los prejuicios que causaban marginación y exclusión; ya no hay razones para la enemistad. Ha quedado radicalmente superado el viejo esquema que imponía favoritismos y acepción de personas y pueblos: somos todos un solo hombre nuevo, plenamente reconciliados y en armonía, porque Cristo "vino a anunciar la paz: paz a ustedes que estaban lejos, y paz a los que estaban cerca. Por Él, unos y otros tenemos libre acceso al Padre en un mismo Espíritu" (Ef 2,17). La mentalidad que seguía criterios mezquinos de división y exclusión, que conducía a despreciar y confrontar, en Cristo ha llegado a su fin (Fitzmyer, 1975, 124). Ahora: "ya no hay judío ni griego, ni esclavo ni libre; ni varón ni mujer, ya que todos ustedes son uno en Cristo Jesús" (Gal 3, 28);

Cristo ha creado "un hombre nuevo" por encima de judíos y griegos y los ha reconciliado con Dios en un solo cuerpo. Por la cruz han cesado las hostilidades, y Cristo ha traído la "paz" (eirene) a los hombres: "Puesto que estamos justificados, tenemos paz con Dios". (Rm 5, 1)

A partir de esta certeza, cobran más sentido las abundantes exhortaciones de Pablo a sus comunidades, para que conserven y fomenten la paz, no solo al interior de la Iglesia, sino también con los de fuera, pues como quedó dicho, ya no hay forasteros ni extraños. Para ser coherentes con la fe cristiana, la paz, que es don del Resucitado y fruto del Espíritu Santo (cf. Rm 8, 6; Gal 5, 22s), debe regir las relaciones interpersonales de los creyentes, de modo que se viva en armonía y fraternidad universal, pues "el Reino de Dios es justicia, paz y alegría en el Espíritu Santo" (Rm 14, 17; Manns, 2010, 935). No por casualidad, "gracia y paz" era no solo un augurio en el saludo inicial de todas las cartas paulinas, sino un verdadero compromiso y programa de vida, en el cual confluían los ideales de bienestar, tanto del pueblo judío como de los pueblos paganos grecorromanos del tiempo de Pablo (cf. 1 Tes 1, 1; 5, 23; Gal 1, 3; 6,16; Filp 1, 2; 4, 9; Film 4; 1 Cor 1, 3; 16, 11; 2 Cor 1, 2; 13, 11; Rm 1, 7; 16, 20$) 7$.

\section{Implicaciones en el campo social}

Si bien reconciliación y paz son, ante todo, fruto y efecto de la obra salvífica de Cristo, y, por lo tanto, en perspectiva paulina, tienen una profunda significación religiosa y teológica, no por ello dejan de tener claras e importantes

7 Se ha discutido mucho sobre el trasfondo y sentido del concepto paz en el augurio del saludo típico paulino, preguntándose hasta qué punto haya sido heredado por Pablo de la shalom bíblica, o haya sido más bien inspirado en el uso que se hacía de esta palabra en el contexto grecorromano. A este respecto, Porter afirma a manera de conclusión: "Una explicación más plausible es que Pablo haya creado intencionalmente su propio encabezamiento [de las cartas] para poner en evidencia la obra universal de Dios, la cual consiste en un don gratuito y en el perdón de la hostilidad precedente. Esto está en perfecta sintonía con el uso que Pablo hace del lenguaje de 'paz' en sus escritos principales, en los que habla de reconciliación, y refleja un trasfondo griego que en este caso presupone un estado objetivo de paz con Dios" (1999, pp. 1129-1130). 
repercusiones en el ámbito de la vida civil y social. Al morir en la cruz, Cristo libera al pueblo judío de la esclavitud respecto de la Ley, y a los pueblos paganos los libera de los límites que impone aquel tipo de sabiduría puramente humana, que a la larga favorece la idolatría; por eso, la cruz termina siendo el signo por excelencia de la reconciliación entre judíos y paganos, y, por lo tanto, símbolo de la reconciliación universal (cf. Ef 2, 11-18; Col 1, 20-23; 3, 15).

En la tradición judía, tanto reconciliación como paz eran valores ideales muy apreciados, pero no siempre reflejados en la realidad, y mucho menos respecto de los pueblos extranjeros. Si en la práctica se procuraba conservar la paz y la armonía al interno del pueblo elegido, hacia los gentiles o paganos prevalecía, más bien, una actitud de abierta hostilidad y confrontación. Los límites del deber de buscar la paz y la reconciliación eran los límites del territorio nacional, abarcando al máximo a los judíos de la diáspora. Ahora bien, en el contexto del Imperio romano, las cosas no eran menos desalentadoras. Sí había una búsqueda de la paz en el Imperio (pax romana), con ciertas coincidencias y semejanzas respecto de la paz cristiana, pero también con grandísimas y radicales diferencias.

La diferencia esencial entre el programa de César y el de Cristo es
la que existe entre una paz impuesta mediante la victoria a través
de la violencia y una paz que se genera a través de una justicia no
violenta. Las dos prometen la paz en la tierra, por lo que la confron-
tación se realiza en el orden de los medios, no de los fines - aunque
ciertamente, unos medios diferentes también cambiarían aquellos
fines comunes-... César no solamente proclama sino que perso-
nifica la paz mediante la victoria violenta, así como Cristo no sólo
proclama sino que también personifica la paz mediante la justicia
no violenta. La teología imperial romana afirmaba que habría paz
en la tierra cuando todo estuviera tranquilo y en orden. En cambio,
la teología cristiana de Pablo sostenía que habría paz en la tierra
cuando hubiera realmente justicia. (Borg y Rossan, 2009, p. 130)

Sería equivocado afirmar que la paz cristiana entendida y propuesta por Pablo se queda en el plano puramente espiritual y religioso, a manera de una virtud limitada al ámbito intimista de la conciencia. La paz verdadera, como la ha comprendido y enseñado el apóstol, necesariamente debe tener repercusiones y proyecciones en el plano social, e incluso político de la vida del creyente. No se trata de un pasivismo resignado frente a las circunstancias del entorno social; todo lo contrario, mueve al compromiso por la justicia, por la creación de las condiciones propicias para la reconciliación y la fraternidad. Y esto no es invención ni capricho de Pablo, sino la consecuencia lógica de hacer derivar la paz y la reconciliación cristianas del misterio pascual de Cristo; en efecto, la muerte en cruz de Jesús tuvo en el contexto romano un claro significado político:

Como medio de punición capital para crímenes horrendos, la crucifixión era la "suprema pena romana", pero casi siempre infligida a la clase inferior (humiliores); la clase superior (honestiores) podía contar con penas más "humanas" (como la decapitación). 
La crucifixión era "la punición típica para esclavos", practicada sobre todo "como escarmiento contra sedición", siendo el ejemplo más espectacular la crucifixión de seis mil seguidores del esclavo rebelde Espartaco en el 71 a. C. (Elliott, 1997, p. 128)

Si la crucifixión en el Imperio romano era un castigo reservado a sedición o delitos considerados de alta traición, e impuesto precisamente para garantizar "la paz y la seguridad", 8 se entiende por qué la paz fundada en un Crucificado tiene en Pablo tanta connotación social y política, y por qué también se atreva a escribirles a los Corintios, que eran una colonia romana: "Cuando fui donde ustedes, hermanos, no fui con el prestigio de la palabra o de la sabiduría a anunciarles el misterio de Dios, pues no quise saber entre ustedes sino a Jesucristo, y éste crucificado" (1 Cor 2, 1-12).

\section{Actualidad y aplicación}

Con las reflexiones que hemos venido haciendo queda suficientemente claro el carácter actual del tema tratado, y además lo urgente que es, para los cristianos de hoy, asumir la reconciliación y la paz como distintivos de nuestra fe y como compromiso inherente a nuestro seguimiento de Jesús. Él fue el Mesías que murió ajusticiado como malhechor en el suplicio de la cruz, y que precisamente desde esa cruz ofreció vida nueva y una propuesta de realización personal y social, radicalmente diversas, respecto tanto de las propuestas legalistas de los judíos como de los ideales romanos, de una paz impuesta por la violencia de la espada.

La realidad de nuestro continente, y de nuestra Nación en particular, reclama de los cristianos coherencia y credibilidad. No basta con declararnos partidarios de la paz, no basta con manifestar que apoyamos procesos sociales de reconciliación y de paz: si de verdad queremos ser consecuentes con nuestra fe cristiana, no podemos sustraernos al compromiso de propiciar las condiciones de justicia que hagan posible esa paz. Además, si bien es cierto que todo camino de reconciliación en perspectiva cristiana debe pasar por el perdón y la misericordia, no por ello debemos aceptar propuestas facilistas que reducen el concepto del perdón a un simple olvido y superación de las ofensas o atropellos sufridos. La fe cristiana, en la perspectiva paulina, nos invita a realizar serios discernimientos de la realidad, para ser propositivos y activos en esa búsqueda de la anhelada armonía entre personas y grupos sociales.

8 A este respecto, resulta interesante el comentario de Neill Elliott $(1997,249)$ respecto a una posible connotación política, casi subversiva, en confrontación con la "pax et securitas" romana de la afirmación que encontramos en 1 Tes 5, 3-6, donde el Apóstol dice: "Cuando digan 'paz y seguridad' entonces mismo, de repente, vendrá sobre ellos la ruina, como los dolores de parto a la que está encinta; y no escaparán...". 
Debemos reconocer que en estos temas, a los cristianos con frecuencia nos asaltan, al menos, dos tentaciones: la indiferencia y la ingenuidad. La indiferencia, cuando creemos, erróneamente, que nuestra fe no tiene nada qué ver con las cuestiones sociales y políticas, y que no tiene nada qué decirle a las problemáticas y conflictos que afectan a nuestros pueblos; y la ingenuidad, cuando pensamos que todas las personas tienen buenas intenciones y no desarrollamos una conciencia crítica cristiana que nos lleve a cuestionar los procesos y decisiones en el ámbito civil, para desenmascarar los intereses y pretensiones contrarios a la voluntad de Dios, que quiere justicia, dignidad y vida plena para todos sus hijos. Pablo tiene muy claro que el ser humano no es una naturaleza abstracta, alejada del mundo, sino un ser profundamente encarnado e inserto en la historia concreta, y que por lo mismo debe asumir compromisos históricos, especialmente en temas de justicia, reconciliación y paz, desde su fe en Cristo (Pastor Ramos, 1999, pp. 491-497).

Los discípulos misioneros de Cristo tenemos que hacer escuchar nuestra voz en los ámbitos gubernamentales donde se toman las decisiones que afectan la marcha pacífica, serena y justa de la vida de nuestra gente. Desde el Evangelio de Cristo, y especialmente como lo interpretó y difundió por todo el mundo el apóstol Pablo, debemos y podemos ofrecer una alternativa de reconciliación y paz que tenga en cuenta la dignidad y los derechos de las víctimas, de los más vulnerables y marginados de la sociedad. La reconciliación que conduce a la paz verdadera no puede lograrse a espaldas y en detrimento de sus reclamos de justicia y reparación. No se puede perder de vista que también la creación (nuestra casa común) ha sido lesionada y ultrajada, y aguarda resarcimiento, respeto y sinceros procesos de reconciliación. ${ }^{9}$

Así como la crucifixión de Jesús, ajusticiado como rebelde político, sirvió de pretexto para salvaguardar la ley y el orden del Imperio, y para defender los intereses de las autoridades judías de entonces, que veían en las palabras y actitudes de Jesús una amenaza a su poder y estabilidad, también hoy existen muchos intereses mezquinos en juego, incluso cuando se habla de búsqueda de reconciliación y paz. Nuestro compromiso cristiano nos empeña en la denuncia de esos propósitos perversos que muchas veces se esconden tras las aparentes buenas intenciones que pretenden perdón y olvido. Siguiendo el ejemplo de Cristo, también nosotros debemos ponernos en el lugar de las víctimas, como deliberadamente lo hizo Pablo. La centralidad del Crucificado en el anuncio del Evangelio paulino es expresión de la opción por las víctimas; desde su dolor, pero también desde sus ilusiones y esperanzas, debemos tomar partido por la justicia, para que haya auténticos procesos de reconciliación y se lleguen a instaurar ambientes de paz estable y duradera (Elliott, 1997, pp. 260-267).

9 La carta Encíclica Laudato Si' del Papa Francisco, nos ayuda a entender las implicaciones del compromiso cristiano en el ámbito ecológico, y la inseparable conexión que existe entre los temas de justicia social, paz y reconciliación, con el respeto y la defensa de los valores ecológicos, para salvaguardar la casa común. 


\section{Referencias}

Borg, M. J. y Rossan, J. D. (2009). El primer Pablo. Estella: Verbo Divino.

Elliott, N. (1997). Libertando Paulo. A justiça de Deus e a política do Apóstolo. Sao Paulo: Paulus.

Fischer, G. (2010). “Riconciliazione (AT)”. En R. Penna, R. Perego y G. Ravasi (eds.), Temi Teologici della Bibbia (pp. 1152-1154). Cinisello Balsamo: San Paolo.

Fitzgerald, J. (2010). “Riconciliazione (nt)”. En R. Penna, R. Perego y G. Ravasi (eds.), Temi Teologici della Bibbia. Cinisello Balsamo: San Paolo.

Fitzmyer, J. (1975). Teología de San Pablo. Madrid: Cristiandad.

Kittel, G., Friedrich, G., y Bromiley, G. (2003). Compendio del Diccionario Teológico del Nuevo Testamento. Michigan: Libros Desafío.

Luján, J. (1991). Concordancias del Nuevo Testamento. Barcelona: Herder.

Manns, F. (2010). “Pace”. En R. Penna, R. Perego y G. Ravasi (eds.), Temi teologici della Bibbia. Cinisello Balsamo: San Paolo.

Martin, R. P. (1999). “Centro della teologia di Paolo". En G. F. Hawthorne, R. P. Martin y D. G. Reid (eds.), Dizionario di Paolo e delle sue lettere. Cinisello Balsamo: San Paolo.

Pastor Ramos, F. (1999). “Ética”. En F. Fernández Ramos (dir.), Diccionario de San Pablo. Burgos: Monte Carmelo.

Porter, S. E. (1999). “Pace, riconciliazione”. En G. F. Hawthorne, R. P. Martin y D. G. Reid (eds.), Dizionario di Paolo e delle sue lettere. Cinisello-Balsamo: San Paolo. 